\title{
Espacio político, territorial y social en los regionalismos Una aproximación al recorrido del Mercosur
}

\section{Political, territorial and social space in regionalisms}

\author{
An approach to the path of Mercosur
}

Lincoln Bizzozero*

\begin{abstract}
Resumen: El trabajo analiza los contenidos que toma el espacio político, territorial y social en el Mercosur. Se parte del supuesto que el proceso de mundialización trae consecuencias en los Estados y también en las regiones. Las consecuencias se plantean en la dimensión política, social y territorial. El regionalismo es una respuesta de los Estados que a su vez cumple un papel específico y genera un ámbito diferenciado. Se analizan tres momentos en función del contexto internacional y de la variable doméstica. Para el contexto internacional se considera principalmente las iniciativas de Estados Unidos. En cuanto a la variable doméstica se consideran los actores y en particular los Estados en su proyección regional. Se exhibe en el trabajo la interrelación entre el proceso mundial, el contexto político internacional, el regional y el nacional.

Palabras-clave: Mercosur. Espacio regional. Dimensión político-social.

Abstract: The paper analyzes the contents that political, territorial and social space takes in the Mercosur. The so-called globalization process has consequences for the states and the regions and affects their political, social and territorial dimension. Regionalism is a response of the states that has a specific role and generates a differential field. The text analyzes three specific moments in Mercosur trajectory according to the international context and domestic variables. For the international context, mainly United States initiatives are considered. As domestic variables, different actors and in particular the states in their regional projection are analyzed. The text highlights the interrelation between the global process and the international, regional ans national political contexts. Keywords: Mercosur. Regional space. Political and social dimension.
\end{abstract}

\footnotetext{
*Doutor em Ciência Política pela Universidade Livre de Bruxelas (ULB, Bruxelas, Bélgica), investigador nível II do Sistema Nacional de Investigadores no Uruguai, professor do Instituto Artigas de Serviço Exterior e da Universidade da República do Uruguai (Udelar), Montevidéu, Uruguai.<lincoln.bizzozero@cienciassociales.edu.uy>. Agradezco los comentarios de los dos árbitros que leyeron este artículo. Sus sugerencias me sirvieron para buscar mejoras en la presentación.
}

Civitas, Porto Alegre, v. 16, n. 3, p. 437-454, jul.-set. 2016 


\section{Mundialización y configuración de nuevos espacios territoriales y sociales}

La década de los noventa del siglo pasado trajo aparejado dos cambios mayores que prefiguraron las condiciones sobre las cuales continuó el proceso de mundialización económico. En primer lugar, el derrumbe del muro de Berlín, implicó la disolución de la Unión Soviética y el cambio de régimen en los países del socialismo europeo. El segundo cambio, que estuvo vinculado a esta modificación en el sistema internacional, fue la incorporación plena e inmediata de estos países a la economía de mercado, a lo cual se agregó durante la década del noventa de manera más administrada, las de la República Popular China y de la India.

Estos dos fenómenos, uno en el orden económico y el otro en el político, pautaron el final del siglo 20 y con ello anticiparon las bases de un nuevo mundo en el siglo 21. Si bien los dos fenómenos están intrínsecamente ligados, el económico asociado a la mundialización del capitalismo prefigura y condiciona las opciones de los estados y demás actores en las definiciones y posiciones frente a distintos temas que han ido surgiendo. De esta manera, se fueron construyendo distintas respuestas de los estados de adaptación a las nuevas realidades y de inserción política y económica al sistema internacional. Estas respuestas no fueron unívocas y tuvieron componentes estructurales vinculados a la posición de cada país en el sistema internacional. Sin embargo tuvieron como componente básico la internacionalización de las economías, lo cual incluyó una mayor o menor apertura al mundo.

La mundialización planteó una necesaria adaptación de los estados frente a la erosión de las fronteras, la reconfiguración de territorios y una nueva percepción del entorno ambiental y del horizonte político y social (Badie, 1995; Badie y Smouts, 1999). La adaptación de los estados incluyó un nuevo equilibrio con la sociedad civil a los efectos de legitimar los ajustes y cambios requeridos por el proceso de inserción internacional. Que los estados hayan accedido a sentar las bases y participar de esta manera del proceso de mundialización hizo posible el ingreso de las sociedades nacionales en un mundo post-nacional, aunque mediado por los estados como conductores de las mismas. Esta mediación parte del vínculo de las sociedades (nacionales) con los estados y del reconocimiento internacional de los mismos, que garantiza esa representación.

La mediación estatal del proceso abre potencialmente la posibilidad de llegar a construir desde los distintos estados un estado mundial cosmopolita, 
o bien llegar a un fracaso en esa construcción con una fragmentación de la sociedad mundial en múltiples regiones (Vandenberghe, 2011).

Lo que importa es que la globalización (que contiene los múltiples aspectos y derivaciones de la mundialización) apareja repercusiones y respuestas de los estados, que incluyen el regionalismo y que atañen la sociedad civil (Serbin, 2005). En esta perspectiva, desde fines del siglo pasado, el proceso de mundialización ha impulsado distintos procesos y sus correspondientes controversias sobre el estado, la sociedad civil, al vínculo entre ambos, la soberanía, el regionalismo, los procesos de regionalización. Todos estos debates deben sumarse al fundamental de la política exterior de los estados que atañe las respuestas que debe plantear frente a los diferentes procesos del sistema internacional y mundial. En estas distintas respuestas que tienen un componente geopolítico y geoeconómico, los estados han propulsado, entre otras, políticas regionales a los efectos de dar contenidos y orientación al regionalismo.

Hay que entender el aspecto fundamental en la mundialización que atañe la reconfiguración de los espacios a partir de la desestructuración y reordenamiento de la estructura productiva y la globalización financiera llevadas adelante principalmente por las empresas transnacionales, acompañadas necesariamente por los bancos y agentes financieros (Piketty, 2014). En la reconfiguración de los espacios participan los estados, ya sea en el nivel doméstico sub-nacional otorgando respuestas nacionales y locales, ya sea en el ámbito regional (cooperación, interdependencia, infraestructura, fronteras), en el continental o mismo en el internacional y mundial (objetivos de desarrollo del milenio, programas de desarrollo, cambio climático).

Este trabajo parte de algunas consideraciones que han sido señaladas anteriormente sobre el papel del estado en el regionalismo para dar contenidos estratégicos a los procesos de regionalización. Estos contenidos no son los mismos para los diferentes estados por lo que los regionalismos tendrán diferentes expresiones como manifestación de distintos modelos (Bizzozero, 1999; 2005; 2012). Estos distintos modelos de regionalismos surgen de las diferentes posiciones de los estados en la estructura del sistema internacional, de los antecedentes históricos de los estados de cada región con el entorno, de los valores culturales en el sistema político y la sociedad civil. Lo que importa considerar es que en esta etapa de transición post-estado-nación, algunos estados tienen proyección internacional y mundial desde la región y a su vez las regiones buscan consolidar posiciones desde el espacio geográfico, como manifestación geopolítica y geoeconómica. De esta manera el regionalismo conforma una respuesta política de los estados no solamente como instrumento 
para la inserción internacional, sino también como modelo de gobernabilidad política, de construcción de la dimensión social y de generación de respuestas políticas desde la región a los procesos de internacionalización y mundialización (Hettne, 2002; Higgot, 1997; Sachwald, 1997).

Cuando se señala que los regionalismos conforman respuestas desde la región a los procesos de internacionalización y mundialización, de alguna forma se señala que son los actores y principalmente los estados como unidades básicas representativas del espacio territorial, los que están dando un diseño y contenidos a la región. Esto implica otras cuestiones que van más allá de la repetición a escala regional de la "voluntad de los estados". En definitiva, las instancias regionales que se generan así como las prácticas, funcionamiento e instituciones son algo novedoso en términos de lo conocido hasta entonces en derecho y en política internacional. El regionalismo es en sí un fenómeno que trasciende al estado-nación, que marca un nuevo horizonte territorial y social, una dinámica institucional y política diferente y otras modalidades de articulación y participación entre los actores del proceso regional. Esto es lo diferente y novedoso del nuevo regionalismo y por eso se diferencia de los anteriores procesos de integración regional, aun cuando el regionalismo pueda englobar y retomar los mismos.

De esta manera, el regionalismo constituye en sí un objeto de análisis en la medida que trasciende al estado-nación y a los propios actores de la sociedad civil en su papel en el territorio nacional. Las estructuras concebidas, los contenidos normativos, las prácticas y funcionamiento, si bien tienen un componente importante de diplomacia inter-estatal no se limitan a ella. Las subregiones de cada estado toman otra dimensión geoeconómica y geopolítica en el proceso regional y pueden incidir de diferente manera en el regionalismo en construcción. A su vez los distintos actores se posicionan de otra manera, en buena medida como resultado de las competencias que les son asignadas por los órganos decisorios del proceso regional.

Como los regionalismos son en sí respuestas de los estados en el marco del proceso de globalización/mundialización/regionalización, uno de los puntos de partida es la relación entre el contexto internacional y el regional. En otros términos, entre los centros y estados impulsores de la globalización y las consecuencias concretas a través de los contenidos políticos en la respuesta de los estados de la región a ese impulso. Cuando se señala los contenidos de la respuesta de los estados, de alguna manera se hace referencia no solamente al núcleo duro de la diplomacia estatal, sino también a los componentes domésticos de las prioridades de la agenda y sobre todo a la participación de la socie-dad civil en su conjunto (mundo político, empresarial, cultural, trabajo, entre otros). 
Este trabajo tiene por objeto visualizar la especificidad de las distintas etapas del Mercosur en la construcción del espacio trans-regional en su dimensión política, territorial y social. Se entiende que los contenidos otorgados a esa construcción por parte de los estados y el vínculo con la sociedad civil es función de dos variables: la externa de los centros impulsores de la globalización que pautan las directrices fundamentales sobre las cuales orientar a los estados; y la doméstica regional que muestra la evolución de la relación estado-sociedad civil. Como la variable externa y en particular las relaciones con Estados Unidos han condicionado en América Latina las respuestas regionales, pueden considerarse básicamente tres instancias desde los inicios del Mercosur hasta la segunda década del siglo 21. La primera instancia se dio en la década del noventa del siglo pasado que coincidió con un impulso a la regionalización con proyección global desde el centro del sistema y en particular de Estados Unidos para los países latinoamericanos. La segunda instancia, que abarcó básicamente la primera década del siglo 21, coincidió con el cambio de prioridades y consecuente retraimiento de Estados Unidos del espacio interamericano. Finalmente, el tercer período, se está procesando actualmente y coincide con el inicio de negociaciones para la conformación de mega-espacios transregionales impulsadas por Estados Unidos (transatlántico y transpacífico).

Estos tres períodos pautan diferentes construcciones del espacio transregional: en el primer y tercer período con fuerte impulso externo ya sea a la regionalización continental (primero) o a la atracción-inclusión en mega/acuerdos (tercero). En el segundo período, en cambio, lo que prima es una reapropiación del territorio político y social en la construcción del espacio regional (Riggirozzi, 2012). Como este segundo período está marcado por las prioridades de la agenda regional, el espacio a construir y a darle contenidos es el mercosuriano-sudamericano, ampliándose posteriormente al latinoamericano/caribeño. También los tres períodos marcan diferencias en relación al papel del estado y como consecuencia en los vínculos con la sociedad civil (y el mercado). El liberalismo de la primera etapa dará mayores márgenes de expresión a la sociedad civil, mientras que la crisis social y económica que se expresa entre fines de la década del noventa y principios del siglo 21 dará lugar a un mayor protagonismo del estado y por ende a un mayor intervencionismo y regulación, lo cual deriva en una disminución de la iniciativa de la sociedad civil (Serbin, 2011).

Esta división en tres períodos correspondientes a los cambios en la política internacional de Estados Unidos en las relaciones con el espacio latinoamericano puede resultar un poco acotada, pero ayuda a visualizar los cambios sistémicos 
que se han ido produciendo en el regionalismo latinoamericano y en particular en el Mercosur. Desde la perspectiva del bloque, la década del noventa del siglo pasado incluyó las etapas de transición y consolidación del Mercosur; durante la primera década del siglo 21 transcurrió la etapa de relanzamiento y se forjó el Programa de Trabajo 2004-2006, que se cerró en los primeros años de la segunda década del siglo 21 con la aprobación del plan estratégico de acción social y el estatuto de la ciudadanía; y finalmente el momento actual en que las negociaciones para la conformación de mega-bloques ha introducido en la agenda regional y nacional el tema de la inserción externa.

El trabajo continuará con el análisis de estos tres momentos de la siguiente manera: el próximo apartado expondrá los contenidos otorgados al regionalismo en los inicios del Mercosur, que explica en parte algunos desarrollos en la participación de la sociedad civil. El siguiente punto se concentrará en la etapa que algunos analistas describen como post-neoliberal y que puso el énfasis en contenidos estratégico-territoriales y sociales. Finalmente, se plantearán los condicionantes aparejados por el contexto internacional con la negociación de mega-acuerdos comerciales (trans-Pacífico y trans-Atlántico). Este contexto retoma el impulso de la década del noventa por lo que necesariamente se cuestionan algunos de los avances registrados durante el siglo 21, al menos los que tienen un componente estatal-nacional y regulador por parte de los estados.

\section{Los inicios del bloque: respuesta de inserción global-regional con oportunidades de expresión liberal de la sociedad civil}

América Latina por su posición en la periferia del sistema internacional no participó del impulso a la regionalización. Por otra parte, los países del Mercosur tenían como una de las prioridades de su agenda la deuda externa y eso manifestaba la vulnerabilidad y limitaciones para encarar los cambios que se iniciaron con la década de los noventa. El estado manifestaba profundas raíces centralistas, burocráticas y autoritarias como consecuencia de procesos dictatoriales en muchos países y de articulación con sectores industriales dependientes del mercado nacional, lo cual no se condecía con las transformaciones aperturistas y críticas de la mundialización/globalización (Negri y Cocco, 2006, p. 162). Además, los sectores sociales habían dejado de visualizar al estado como un posible articulador nacional y promotor del desarrollo, debido entre otras cosas a la percepción negativa referida a la responsabilidad que le cabía en el incremento de la deuda externa, la gestión económica, el aumento de las desigualdades sociales. Finalmente, en el plano de las ideas, el modelo de sustitución de importaciones y las recetas de la Cepal 
para remediar las consecuencias del desarrollo desigual fueron dejados de lado y cuestionados en los circuitos de intermediación y difusión de expertos internacionales y entre la mayoría de los analistas académicos.

El programa de reformas del "Consenso de Washington" constituyó un marco de referencia para los países de la región. El eje común de los países de América Latina estuvo signado por la apertura económica, la disminución del papel del estado en la economía y en la articulación de los sectores sociales. Los temas referidos al papel del estado en la economía y sociedad, a la apertura económica y comercial y a los cambios institucionales derivados de la disminución de las atribuciones estatales posibilitaron una agenda interamericana positiva con Estados Unidos.

Todo esto fue canalizado en el programa de algunos partidos políticos de credo liberal, que supieron interpretar el programa del "Consenso de Washington" y transmitirlo en las respectivas sociedades nacionales de los países que iniciaron el Mercosur. De hecho, los gobiernos que firmaron el tratado de Asunción tuvieron coincidencias de base en la concepción liberal de las reformas, que posibilitaría un mejor posicionamiento en el mundo. La correspondencia de un estado menos regulador requirió necesariamente de una sociedad civil más protagonista, para dar consistencia y legitimidad a los procesos regionales, incluyendo los que se iniciaron en la década del noventa del Alca y del Mercosur con la Unión Europea (Tussie y Botto, 2003).

La participación de la sociedad civil se fue dando paulatinamente de acuerdo a la distancia de los diferentes actores de los órganos de decisión regional (Consejo Mercado Común y Grupo Mercado Común) y de los intereses económicos concernidos. Sin embargo, algunos actores sociales estuvieron, desde los inicios del proceso regional, activos, vigilantes y con un grado de incidencia como fue el caso de las centrales sindicales del Cono Sur. Otras organizaciones sociales vinculadas a temas económicos como ser los industriales y empresarios y a temas sensibles como la educación, la salud sanitaria y la salud humana fueron estructurando comunidades de interés regional. El mapa de participación de la sociedad civil se fue diseñando durante la década del noventa mostrando un creciente interés y cambios en la burocracia y decisiones del estado en relación a la articulación estado-sociedad civil. Se comenzó a visualizar el surgimiento de una incipiente sociedad civil regional que de alguna manera modificaba las bases de funcionamiento estatal (Grandi y Bizzozero, 1997).

Se puede rastrear la creciente participación de actores de la sociedad civil en el Mercosur durante la década del noventa en las etapas de transición primero y consolidación después. En la etapa de transición, la leve estructura 
institucional posibilitó un derrame institucional y participativo, que generó distintas ramificaciones e inclusión de actores. La inclusión del foro consultivo económico y social entre los órganos en el protocolo de Ouro Preto confirmó el necesario equilibrio entre los estamentos burocrático-estatales y actores de la sociedad civil. Posteriormente el surgimiento de la red de Mercociudades dio pie a nuevas iniciativas y generación de programas en el marco de la red, que propulsó algunas de ellas en el bloque regional, en las regiones y ciudades (Bizzozero, 2003). Todo esto sin contar que el entramado institucional del Mercosur creció y posibilitó también la inclusión, consulta y participación de actores en los respectivos sectores involucrados. Es claro que la propia estructura institucional decisoria del Mercosur continuó sin modificarse por lo que las iniciativas que se fueron gestando quedaron entrampadas en el cuello de botella resolutoria del Grupo Mercado Común.

Las iniciativas continentales de Estados Unidos y la gestación de un espacio inter-bloques UE-Mercosur propulsaron aún más esta dinámica participativa y legitimadora de los actores de la sociedad civil. Esto fue así porque en ambas negociaciones, con algunas diferencias, se otorgó a la sociedad civil un lugar específico de consulta y confirmación en los avances de las negociaciones. El lugar que se les otorgó a los representantes de la sociedad civil en esas instancias, se debió fundamentalmente a los requerimientos legitimadores de las organizaciones de derechos humanos, sindicales y ambientales de Estados Unidos y europeas que buscaban una contraparte social que pudiera hacer el equilibrio necesario frente a la expresión política de los gobiernos.

La iniciativa de las Américas que propulsó el presidente de los Estados Unidos, George Bush, en 1990, intentó ser una respuesta en distintos frentes a los cambios aparejados por la caída del muro de Berlín y el proceso de mundialización/globalización. Por una parte el presidente de los Estados Unidos planteó la posibilidad de un acuerdo para el conjunto de las Américas, a los efectos de conformar una sociedad común, en lo que constituiría el primer continente libre del mundo y también de la historia (Tulchin, 1992, p. 39). En ese sentido, los temas económicos y el (libre) comercio quedaron planteados con una visión estratégica y conformaron el núcleo duro de la Iniciativa y del eje articulador para nuclear a toda la región de las Américas y del Caribe con una identidad común frente al resto del mundo.

La Iniciativa para las Américas tuvo una respuesta positiva de los países de la región, pero fue rápidamente suplantada por una propuesta más ambiciosa, acorde a los resultados de la Ronda Gatt de Uruguay y la puesta en marcha de la Organización del Comercio. Esa propuesta incluyó una parte política, otra de cooperación y finalmente la más sustantiva, el denominado 
Acuerdo de Libre Comercio de las Américas (Alca), que impulsó desde 1994 el presidente Clinton, en el marco de los nuevos temas de la agenda de comercio internacional. Es frente a esta propuesta y frente al despliegue de las distintas prioridades por parte de Estados Unidos en relación a los países de América Latina, que se plantearon diferentes respuestas que irán marcando el mapa de las relaciones interamericanas en la década de los noventa.

En el caso de los países del Mercosur, paralelamente al Alca se fueron desarrollando negociaciones con la Unión Europea con vistas a crear el primer acuerdo interregional de libre comercio en el sistema de comercio internacional. Las negociaciones UE-Mercosur en el Acuerdo Marco Interregional (AMI) se plantearon sobre la base de tres pilares: un acuerdo político, uno de cooperación y finalmente un acuerdo de libre comercio. Lo que importa señalar es que el núcleo duro de la negociación estuvo centrado sobre todo en el tercer pilar, en los temas agrícolas y de servicios, aun cuando algunos temas políticos mostraron las debilidades de esta articulación atlántica con vistas a generar un nuevo escenario en el sistema internacional.

En la medida que la agenda regional estuvo pautada por temas impulsados desde el exterior por Estados Unidos y la Unión Europea y mostró una convergencia de perspectivas, aun limitado por las asimetrías en el desarrollo y por importantes divergencias en temas sensibles, se comenzó a hablar de un triángulo Occidental (Grandi, 2000). La percepción del espacio político y territorial estuvo condicionada por la evolución de la agenda externa y las negociaciones que llevó el Mercosur en diferentes ámbitos (OMC, Alca, AMI). A su vez los actores de la sociedad civil comenzaron a demandar una mayor presencia del estado en las negociaciones y de hecho no cuestionaron la regionalización de la agenda que se inició en el año 2000 a instancias del gobierno de Cardoso en Brasil, como consecuencia del estancamiento de las negociaciones del Mercosur en los distintos ámbitos.

En el siglo 21 las prioridades de la potencia hegemónica centradas en otros espacios mundiales, la crisis del modelo neo-liberal, las respuestas estructurales de algunos estados sudamericanos y el cambio en el escenario económico desde mediados de la primera década del siglo 21 plantearon bases para que el estado reasumiera un mayor protagonismo en el ámbito nacional. Estos cambios en el sistema internacional, con el retraimiento de Estados Unidos de la región latinoamericana, en el ámbito nacional con el surgimiento de gobiernos más intervencionistas en las políticas públicas repercutieron en los contenidos del regionalismo que se comenzaron a esbozar en el Mercosur hacia mediados de la primera década del siglo 21. 


\section{El espacio regional en la primera década el siglo 21: un reposicionamiento estratégico del estado}

Los cambios en el contexto internacional signados por el giro de la política internacional de Estados Unidos y la modificación en la estructura económica internacional a partir del crecimiento de la República Popular China, de algunas potencias emergentes y de la recuperación de Rusia, posibilitaron un mayor margen de autonomía en las relaciones políticas y económicas de los países del Mercosur y en general de América Latina. Por otra parte, el estado comenzó a modificar las bases sobre las cuales había edificado una respuesta a los requerimientos de la globalización en la década de los noventa. Sin embargo, no fue un retorno al estado desarrollista, sino una recomposición del estado en su dimensión política, territorial y social. Esta recomposición incluyó la necesaria articulación entre los intereses domésticos nacionales y regionales a los efectos de plasmar en el bloque regional la dimensión política y social, que se consideraba deficitaria en el paradigma del Mercosur comercial.

Esta recomposición del estado se asentó, en los países del Mercosur y algunos latinoamericanos, en la asunción de gobiernos de izquierda o neo-desarrollistas, que cuestionaron el modelo neo-liberal y las recetas implementadas en la política económicas, las políticas sociales y en el modelo de desarrollo. Por otra parte, las agendas domésticas modificaron sus prioridades, incluyendo entre los temas primerizos los político-institucionales y sociales. Como las agendas domésticas fueron de vuelta centradas por los sistemas políticos, el estado recuperó un papel central en las sociedades a partir de la articulación de los intereses y su proyección en el espacio regional e internacional.

La articulación del estado con la región y el espacio global/mundial a partir de las sociedades nacionales debe visualizarse como una respuesta diferencial a la que se diera en los noventa. En el siglo 21 el estado se posicionó estratégicamente proyectando los intereses sociales en el espacio regional y mundial. La región a su vez constituyó una plataforma necesaria para proyectar esos intereses sociales y es a través de ella que el estado pudo dar sustento y contenido a las (nuevas) ideas sobre el regionalismo.

La crisis del modelo neo-liberal facilitó la asunción de gobiernos neodesarrollistas, volcados a la izquierda del espectro político, en los países del Mercosur. El eje estratégico argentino-brasileño se reconfiguró y asumió nuevos contenidos, prioridades y objetivos, en sintonía con los programas y propuestas que encararon Lula da Silva y Néstor Kirchner. Los encuentros 
mantenidos por Lula con Duhalde y Kirchner plasmaron las nuevas realidades, objetivos y propuestas. Esas nuevas realidades se plasmaron como una iniciativa regional y se refrendaron en el Programa de Trabajo 2004-2006. De esta manera, el Programa de Relanzamiento del 2000 y posteriormente, con mayor nitidez y definiciones, el Programa de Trabajo 2004-2006, delinean un camino para ir dando contenidos al espacio transregional en su dimensión político, social y territorial.

Como ya señalara en otros trabajos, la evaluación de las decisiones tomadas por el Consejo Mercado Común durante la década posterior a la aprobación del Programa de Trabajo 2004-2006, da muestras de una mayor politización del bloque regional, expresada en los propios órganos creados, entre los cuales el Parlamento del Mercosur y el cargo de Alto Representante General (Bizzozero, 2013). No fueron esas las únicas concreciones políticas durante la década 2003-2013, ya que se generaron varios órganos vinculados a ámbitos de competencia ministeriales de cada país, en el ámbito del Consejo Mercado Común. En el caso de los ministerios de desarrollo social, se creó un órgano que contempló la dimensión social, pero que a su vez canalizó la articulación con la sociedad civil y los ámbitos generados para la participación vía cumbres sociales.

Para este reposicionamiento estratégico del estado en su dimensión regional-territorial-social fue clave el papel que asumió Brasil en relación a su propio espacio territorial y también en su relación con el resto de países de América del Sur. El reposicionamiento del estado por otra parte posibilitó vincular la dimensión nacional- territorial - social con las regiones fronterizas y la regional-territorial-social. En el caso de Brasil posibilitó otorgar una respuesta político-global a las demandas regionales de seguridad y cooperación y a través de la plataforma regional proyectar un modelo de gobernabilidad mundial. En ese sentido, la política internacional de Brasil es mundial a partir del modelo regional que va forjando a través de una política regional incremental en institucionalidad, cooperación y desarrollo.

El reposicionamiento se inició con Fernando Henrique Cardoso en el año 2000 y se profundizó durante las dos presidencias de Lula da Silva. El componente territorial se manifestó desde la cumbre convocada por Fernando Henrique en el año 2000, donde se creó y se plantearon los cimientos de la Iniciativa para la Integración Regional Sudamericana (IIRSA) en el marco de la primera Cumbre Sudamericana. Este marco regional sudamericano sentó la plataforma para preparar y articular proyectos de infraestructura en transporte, energía y telecomunicaciones transnacionales. De esta manera, las prioridades de infraestructura nacional pudieron tener, en algunos casos, 
una proyección regional. En el caso de Brasil, por su tamaño territorial e importancia estratégica regional, el vínculo nacional-regional con el IIRSA se expresó en el programa de aceleración del crecimiento.

Si el mapa de fines de la década del noventa mostraba un espacio regional con un déficit territorial-regional y social, a inicios de la segunda década del siglo 21 el mapa del Mercosur había alcanzado territorialmente al conjunto de América del Sur independiente. Esto fue así porque todos los estados de América del Sur que no eran parte del núcleo duro del bloque regional firmaron acuerdos por el cual quedaron incluidos en el funcionamiento como estados asociados. Esta imbricación sudamericana de hecho se comenzó a cruzar con el funcionamiento de Unasur, aun cuando quedaron delimitados por los objetivos concretos de cada acuerdo.

Al reposicionarse en el espacio territorial específico y sudamericano, el Mercosur tomó necesariamente una dimensión política y social. El posicionamiento en la dimensión política posibilitó una mayor presencia del bloque regional, sobre todo una vez que se creó el cargo de Alto Representante General. Lo que importa fue el nuevo equilibrio generado entre el estado y la sociedad civil. Si bien se generaron espacios para la expresión de la sociedad civil, al estar demasiado reguladas por los estados respectivos, perdieron el impulso e iniciativa característicos de la década anterior. Por otra parte, la reconfiguración e importancia a los territorios para generar obras e inversiones en infraestructura, derivaron en nuevas situaciones conflictivas con las poblaciones involucradas.

Desde una perspectiva sistémica el reposicionamiento del bloque regional en América del Sur con un alcance en la dimensión política y social, pudo irse concretando por la limitada prioridad otorgada por Estados Unidos a América Latina. El desgaste de las intervenciones en Afganistán e Irak aunado a la crisis económica con la crisis financiera de las hipotecas y la falta de capacidades y recursos para encaminar una salida en distintas cumbres y negociaciones internacionales, plantearon las limitaciones de la política internacional de Estados Unidos y a su vez se perfilaron espacios posibles para ser ocupados y desarrollados en algunas regiones del mundo, entre las cuales América Latina, y en algunos ámbitos e instancias internacionales. Una de las manifestaciones de esta menor presencia de Estados Unidos en la región fue el final con un balance negativo de las negociaciones del Alca.

La importancia asumida por las potencias emergentes (PEMS) como consecuencia de su creciente participación en la economía mundial y en distintos ámbitos y foros de negociación y los límites sistémicos globales trajo a su vez una nueva situación en el sistema internacional. La estructura 
del sistema ha tomado una nueva complejidad de varios niveles en que además del estratégico-político y el económico-político pueden encontrarse niveles atinentes a los límites sistémicos globales (energético, climático, alimentario). Si bien estos niveles están interrelacionados con los otros dos, al tomar esa relevancia sistémica pasan a tener una dimensión propia y específica.

Desde otro punto de vista, lo que importa es que los límites sistémicos y la participación de otros actores en la estructura dan lugar a una articulación en distintos niveles de la misma por parte de diferentes estados y actores, algunos de los cuales se repiten, que pueden generar principios, normas y bases de políticas en los diferentes temas tratados. Esto hace que se deba analizar cada nivel y la articulación entre los distintos niveles para entender el funcionamiento actual del sistema internacional. De esta manera, el regionalismo de la primera década del siglo 21 fue una respuesta sistémica a los requerimientos del sistema internacional y a la limitada presencia de Estados Unidos en América Latina.

Las regiones pueden potenciarse específicamente en alguno de los niveles y/o proyectar solamente la posición del estado-región. En cualquiera de los casos lo que importa es la vinculación entre el nivel regional y el internacional, si el mismo esboza una función de adaptación a los requerimientos internacionales o de participación activa en las definiciones internacionales del nivel analizado. Esta vinculación estado-bloque regional-sistema internacional plantea la potencialidad y límites generados por el bloque regional en su dimensión política, social y territorial. Es en este marco que debe situarse la participación de Brasil en Brics como potencia emergente.

Lo que importa señalar es que el regionalismo de los noventa fue reestructurado a partir de una convergencia de factores entre los cuales primordialmente la pérdida de posición política y económica de Estados Unidos y accesoriamente de la Unión Europea y su repercusión en tanto modelos ordenadores de la gobernanza global, la presencia económica (y no solamente comercial) de China y de una nueva formulación del papel del estado en la política internacional y el desarrollo.

De esta manera, el contexto internacional y los factores domésticos vinculados a una demanda social por un mayor protagonismo y regulación por parte del estado, son factores explicativos que posibilitan entender la vinculación entre las nuevas definiciones del Mercosur a través del relanzamiento y sobre todo del Programa de Trabajo 2004-2006 y los alcances y contenidos de la dimensión política, territorial y social.

Los inicios de la segunda década del siglo 21 mostraron un Mercosur diferente en su dimensión política, social y territorial. Si bien esta nueva 
configuración del Mercosur planteó nuevos objetivos políticos, sociales y territoriales con vistas a darle continuidad al camino trazado, las definiciones del gobierno de Estados Unidos para generar un salto cualitativo en el sistema de comercio internacional con la concreción de mega-bloques regionales, replanteó debates sobre el bloque regional, la inserción internacional, el papel del estado y los vínculos con la sociedad civil.

\section{El Mercosur y el espacio transregional: perspectivas frente a las mega-negociaciones}

Con el inicio de la segunda década del siglo 21, el órgano político del bloque regional tomó decisiones que transparentaron algunos objetivos y definiciones. El Consejo Mercado Común aprobó en el año 2010 el Programa Estratégico de Acción Social, el Estatuto de la Ciudadanía, la creación del cargo de Alto Representante General del Mercosur y el Programa de Consolidación de la Unión Aduanera. Esas cuatro decisiones referidas al ámbito social, el ciudadano, el político y el económico, al inicio de la segunda década del siglo 21, pautan el camino y la orientación del bloque regional.

Además entre el año 2012 y el 2013 el mapa territorial y político del bloque regional fue modificado en su composición nuclear y sus alcances. En el año 2012 el bloque aplicó por primera vez el Protocolo de Ushuaia sobre compromiso democrático en el Mercosur, la República de Bolivia y la República de Chile, suspendiendo la participación de Paraguay. Esta suspensión posibilitó a su vez el ingreso de la República Bolivariana de Venezuela como estado parte, ya que faltaba la ratificación del parlamento paraguayo como último requisito. En julio del 2013, durante la cumbre del Mercosur que se realizó en Montevideo, el bloque concluyó la telaraña de acuerdos con los países de América del Sur, al firmar los acuerdos marcos de asociación con la República de Surinam y la República Cooperativa de Guyana. De esta manera, el Mercosur terminó de completar una dimensión sudamericana entre los estados-parte y los estados asociados y dejó abierta la posibilidad para generar acuerdos en el espacio latinoamericano, lo cual incluiría México, América Central, Cuba, República Dominicana y Haití.

De esta manera el bloque regional inició la segunda década del siglo 21 posicionándose con vistas a continuar el proceso de regionalización de las agendas domésticas nacionales, de continuidad en la construcción de la dimensión política y social y de proyección político-territorial. En este escenario, se continuaba convalidando el regionalismo continental impulsado por Brasil que se fijó como horizonte un pilar político y de cooperación en la región sudamericana con proyección a América Latina/Caribe y uno 
económico en que el estado apoya los emprendimientos empresariales para proyectarse a la región (y al mundo) (Cervo, 2010; Bernal-Meza, 2011).

Algunos cambios que se fueron produciendo en el contexto internacional, entre los cuales el inicio de negociaciones, impulsadas por Estados Unidos, para conformar mega-bloques; la pérdida de empuje de China como motor de la economía mundial; y las consecuencias económicas en los países de América Latina, configuraron un panorama diferente en el regionalismo del bloque regional hacia mediados de la segunda década del siglo 21 .

Las negociaciones para conformar un bloque transpacífico y las que se iniciaron entre Estados Unidos y la Unión Europea con vistas a un acuerdo transatlántico abrieron nuevos debates y controversias en la región sobre la inserción internacional, el papel de los estados y la vinculación con el mercado, las relaciones externas entre otros. Las controversias tuvieron un componente político doméstico en las elecciones que se desarrollaron en distintos momentos y procesos en Argentina, Brasil, Paraguay, Uruguay y Venezuela. Las plataformas electorales incluyeron temas y propuestas vinculadas a las políticas regionales y al regionalismo. Por otra parte, surgieron propuestas regionales en América Latina en el marco del nuevo escenario de mega-acuerdos que buscan canalizar y formalizar las cadenas de valor en distintos sectores industriales. De esta manera, se creó la Alianza para el Pacífico, en abril del 2011, entre Colombia, Chile, México y Perú, que en pocos años generó expectativas en América Latina y en el mundo.

La Alianza para el Pacífico tiene como uno de los pilares claves el de conformarse en tanto instancia regional para no perder determinadas preferencias negociadas en los distintos acuerdos de libre comercio, sea con Estados Unidos, la Unión Europea o bien con algunos países de AsiaPacífico, sobre todo teniendo en cuenta que se están negociando megaacuerdos regionales. Por otra parte, la Alianza para el Pacífico se presentó como propuesta alternativa y al mismo tiempo competitiva con el Mercosur, al menos en los primeros años. En esta propuesta, así como en algunos programas partidarios se plantea una propuesta de regionalismo diferente, centrada más en la libre circulación de bienes, capitales y servicios, con un menor contenido en la dimensión política, social y territorial, espacio que quedaría en el ámbito nacional.

El Mercosur acaba de cumplir veinticinco años desde que se aprobara el tratado de Asunción en 1991. Lo hace en momentos en que se produce una transición en los contenidos a otorgar al espacio político-socialterritorial. El cambio de gobierno en Argentina, la situación en Brasil y la 
propia agenda regional de definiciones sobre las opciones de desarrollo e inserción internacional, dejan abiertas las definiciones en cuanto a los nuevos contenidos del espacio regional en la dimensión política, social y territorial.

El proceso de impeachment en Brasil no se ha completado al escribir estas líneas. Sin embargo, resulta bastante evidente el desacople social del proceso político. Esto lleva a plantear que la nueva configuración del espacio político-social y territorial tendrá un componente de creación social más evidente que lo que fuera la participación de las organizaciones de la sociedad civil en los noventa. Esto se debe al hecho de que hay avances normativoinstitucionales y en capacidades e instrumentos de acción en derechos sociales, ambientales y de participación. En definitiva, se tendrán nuevas expresiones de la sociedad civil atinentes a estos derechos para buscar profundizar los mismos en nuevos marcos regionales.

Esto nos lleva a plantear en nuevos formatos la dinámica articuladora variable externa-variable doméstica: si bien la iniciativa externa continuará marcando y delimitando las opciones estratégicas de los estados en materia de inserción internacional, la variable doméstica tendrá un peso incremental en los alcances y contenidos de la dimensión territorial y social referidos a los derechos humanos, ambientales y sociales.

\section{Conclusiones}

Este trabajo se planteó analizar el Mercosur desde su gestación en lo que concierne los contenidos otorgados al espacio regional en la dimensión policía, social y territorial. Para ello se partió de un marco conceptual en que se otorgan fundamentos sobre la importancia del regionalismo. Como el regionalismo es una respuesta política que han dado los estados para orientar el proceso regional en la mundialización/globalización, se consideró como una de las variables el contexto internacional.

Se consideraron tres momentos claves en función del impulso externo a la globalización, cuyo centro referencial para los países del Mercosur y de América Latina/Caribe es fundamentalmente Estados Unidos. Esos momentos fueron básicamente de impulso en la década del noventa, de retraimiento del espacio regional en la primera década del siglo 21 y de renovación del impulso externo a través de mega-negociaciones.

Los tres momentos plantean diferentes situaciones en la articulación estado-sociedad civil y en la configuración política y social del territorio. A su vez también plantean diferencias en los alcances estratégicos que tienen los estados y sobre todo Brasil una vez que pasa a conformar como potencia 
emergente parte de los Brics y cumple un papel de impulsor de la agenda regional y también sudamericana (y latinoamericana) y de articulador con el sistema internacional.

La etapa que se está transitando abre una nueva instancia de controversias diversas sobre los contenidos del regionalismo en el proceso de globalización. Por un lado, al interior de los estados y de las regiones por el componente estratégico que tienen y por el otro en distintos sectores de la sociedad civil por los alcances y limitaciones que puedan tener en la participación.

\section{Referencias}

BADIE, Bertrand. La fin des territoires. Paris: Fayard, 1995.

BADIE, Bertrand; SMOUTS, Marie-Claude. Le retournement $d u$ monde: sociologie de la scène internationale. 3 ed. Paris: Presses de Sciences Po/Dalloz, 1999.

BERNAL-MEZA, Raúl. El regionalismo: concepto, paradigmas y procesos en el sistema mundial contemporáneo. In: Lincoln Bizzozero; Isabel Clemente (coord.). La política internacional en un mundo en mutación. Montevideo: Banda Oriental, 2011. p. 11-30.

BIZZOZERO, Lincoln. La mundialización de la cuestión laboral y el comercio: respuestas de articulación de los nuevos regionalismos en América del Norte, Europa y el Cono Sur de América Latina. Estudios Internacionales, n. 127-128, p. 3-35, 1999.

BIZZOZERO, Lincoln. La participación de los actores de la sociedad civil en el Mercosur. Hacia una ciudadanía regional en el bloque? In: Andrés Serbin (comp.). Entre la confrontación y el diálogo: integración regional y diplomacia ciudadana. Buenos Aires: Siglo XXI/Universidad de Belgrano, 2003. p. 87-116.

BIZZOZERO, Lincoln. Derechos humanos y dimensión social en los regionalismos del siglo XXI. Construcción y perspectivas desde el espacio regional del Mercosur. In: Políticas públicas de derechos humanos en el Mercosur: un compromiso regional. Montevideo: Observatorio de políticas públicas de derechos humanos en el Mercosur, 2005.

BIZZOZERO, Lincoln. Endebles pilares del regionalismo estratégico: el Mercosur en el inicio de la segunda década del siglo XXI. In: Alfredo Guerra-Borges (coord.). Panorama actual de la integración latinoamericana y caribeña. México: Unam-IIE 2012. p. 51-82.

BIZZOZERO, Lincoln. Estrategia, temas y alcances del Mercosur en el siglo XXI: fotografía de decisiones desde el Programa de Trabajo 2004-2006. Mural Internacional, v. 4, n. 2,p. 48-54, 2013<10.12957/rmi.2013.8369>. <http://www.observatoriomercosur. org.uy/libro/derechos_humanos_y_dimension_social_en_los_regionalismos_del_ siglo_XXI_4.php>.

CERVO, Amado. A internacionalização da economia brasileira. Brasília: Fundação Alexandre Gusmão/Thesaurus Editora, 2010. 
GRANDI, Jorge. Mercosur - TLCAN - UE: triángulo atlántico o triángulo de las Bermudas. Civitas: Revista de Ciências Sociais, n. 1, v. 1, p. 97-122, 2000 $<10.15448 / 1984-7289.2001 .1 .68>$.

GRANDI, Jorge; BIZZOZERO, Lincoln. Hacia una sociedad civil del Mercosur, viejos y nuevos actores en el tejido subregional. Integración y Comercio, n. 3, p. 33-50, 1997.

HETTNE, Björn. El nuevo regionalismo y el retorno a lo político. Comercio Exterior, v. 52, n. 11, p. 954-965, 2002.

HIGGOTT, Richard. Mondialisation et gouvernance: l'émergence du niveau régional. Politique Étrangère, v. 62, n. 2, p. 277-292, $1997<10.3406 /$ polit.1997.4640>.

NEGRI, Antonio; COCCO, Giuseppe. GloAL: Biopoder y luchas en una América Latina globalizada. Buenos Aires: Paidós, 2006.

PIKETTY, Thomas El capital en el siglo XXI. Buenos Aires: Fondo de Cultura Económica, 2014.

RIGGIROZZI, Pia. Re-territorializando consensos: hacia un regionalismo posthegemónico en América Latina. In: Andrés Serbin; Laneydi Martínez; Haroldo Ramanzini Júnior. El regionalismo "post-liberal” en América Latina y el Caribe: nuevos actores, nuevos temas, nuevos desafíos. Anuario de la integración regional de América Latina y el Gran Caribe 2012. Argentina, 2012. p. 129-152.

SACHWALD, Frédérique. La mondialisation comme facteur d'intégration régionale. Politique Étrangère, v. 62, n. 2, p. 257-264, $1997<10.3406 /$ polit.1997.4638>.

SERBIN, Andrés. Globalización, regionalismo y sociedad civil. Documentos Cries, n. $1,2005$.

SERBIN, Andrés. Los nuevos escenarios de la regionalización: déficit democrático y participación de la sociedad civil en el marco del regionalismo suramericano. Documentos Cries, n. 17, 2011.

TULCHIN, Joseph. La iniciativa Bush para las Américas ¿Gesto vacío, hábil estrategia o cambio reseñable en las relaciones continentales? América Latina Hoy - Revista de Ciencias Sociales, v. 4, p. 39-48, $1992<$ http://www.acuedi.org/ddata/94.pdf>.

TUSSIE, Diana; BOTTO, Mercedes (coord.). El Alca y las Cumbres de las Américas: ¿Una nueva relación público-privada? Buenos Aires: Flacso, 2003.

VANDENBERGHE, Frédéric. Um estado para o cosmopolitismo. Novos Estudos, n. 90, p. 85-101, 2011.

VELTZ, Pierre. Une organisation géoéconomique à niveaux multiples. Politique Étrangère, v. 62, n. 2, p. 265-276, $1997<10.3406 /$ polit.1997.4639>.

Autor correspondente:

Lincoln Bizzozero

Luis Piera 1835 ap. 24

11200 Montevideo, Uruguay

Recebido em: 21 mar. 2016

Aprovado em: 22 jun. 2016 